\title{
SOME CONDITIONS FOR FINITENESS OF A RING
}

\author{
HOWARD E. BELL \\ Department of Mathematics \\ Brock University \\ St. Catharines, Ontario, Canada L2S 3A1 \\ (Received May 11, 1987 and in revised form June 24, 1987)
}

ABSTRACT. Extending a result of Putcha and Yaqub, we prove that a non-nil ring must be finite if it has both ascending chain condition and descending chain condition on non-nil subrings. We also prove that a periodic ring with only finitely many non-central zero divisors must be either finite or commutative.

KEYS WORDS AND PHRASES. Finiteness of rings, Periodic rings, Commutativity • 1980 AMS SUBJECT CLASSIFICATION CODES. 16A44, 16A70.

1. INTRODUCTION AND TERMINOLOGY.

Over the years several authors have given sufficient conditions for a ring $R$ to be finite, among them the following:

(I) (Szele, [9]) $\mathrm{R}$ has both ascending chain condition and descending chain condition on subrings;

(II) (Ganesan, [4], [5]) $R$ has non-trivial left zero divisors, of which there are only a finite number;

(III) (Bel1, [1]) $R$ contains no infinite zero ring and no infinite subring without non-zero nilpotent elements;

(IV) (Putcha and Yaqub, [8]) $\mathrm{R}$ is non-nil and has only finitely many nonnilpotent elements.

The present study, which presents some new conditions for finiteness, was motivated by the Putcha-Yaqub paper. Our first two theorems are ones suggested by that paper; the third is a new result on the old theme of commutativity and finiteness.

Throughout the paper the term zero divisor will refer to a one-sided (i.e. not necessarily two-sided) zero divisor. By a left (right) zero divisor we shall mean an element $\mathrm{y}$ for which there exists $\mathrm{x} \neq 0$ such that $\mathrm{yx}=0 \quad(\mathrm{xy}=0)$.

If $x_{1}, x_{2}, \ldots, x_{k} \in R$, the subring generated by the $x_{i}$ will be denoted by $\left\langle x_{1}, x_{2}, \ldots, x_{k}\right\rangle$; and for each $x \in R$, the symbols $A_{\ell}(x)$ and $A_{r}(x)$ will denote respectively the left and right annihilators of $x$. The symbols $C$ and $N$ will be used for the center of $R$ and the set of nilpotent elements of $R$. The symbol $\mathrm{Z}$ will denote the ring of integers, and $\mathrm{Z}^{+}$the set of positive integers.

Finally, the ring $R$ is called periodic if for each $x \in R$, there exist distinct $\mathrm{m}, \mathrm{n} \in \mathrm{z}^{+}$for which $\mathrm{x}^{\mathrm{m}}=\mathrm{x}^{\mathrm{n}}$. 
2. TWO FINITENESS THEOREMS FOR NON-NIL RINGS.

Our first theorem, which employs (IV) in its proof, is an extension of (II).

THEOREM 1. Let $R$ be a ring, and let $S$ be the set of non-nilpotent zero divisors of $R$. If $S$ is finite and non-empty, then $R$ is finite.

PROOF. Let $x \in S$. Applying the pigeonhole principle to the powers of $x$ yields distinct $m, n \in z^{+}$for which $x^{m}=x^{n}$; consequently, there exists a non-zero idempotent zero divisor $e$, which we assume to be a right zero divisor. Write $R=e R+A_{r}(e)$. Since each summand consists of zero divisors of $R$, each has only finitely many nonnilpotent elements, hence by (IV) is either finite or nil. It is immediate that eR is finite, and to complete the proof we proceed on the assumption that $A_{r}(e)$ is $n i l$. Let $0 \neq x \in A_{r}(e)$, with $x^{s}=0 \neq x^{s-1}$. Then $(e+x) x^{s-1}=0$, so $e+x$ is a zero divisor. Moreover, $e+x$ is non-nilpotent, since for any $k \geqq s$, we have $(e+x)^{k}=e+{ }_{i=1}^{k} x^{i} e$; and the assumption that $(e+x)^{k}=0$ gives, on left multiplication by $e$, the contradiction $e=0$. It follows that the set $\left\{e+x \mid x \in A_{r}(e)\right\}$ is finite, hence $A_{r}(e)$ is finite and so is $R$.

THEOREM 2. If $R$ is any non-nil ring having both ascending chain condition and descending chain condition on non-nil subrings, then $R$ is finite.

PROOF. Note that by (I) and (III), any infinite ring $R$ satisfying our hypotheses, and indeed every infinite subring of $R$, must contain an infinite zero ring. Moreover, for any non-nilpotent element $x$, the chain $\langle x\rangle 2\left\langle x^{2}\right\rangle 3\left\langle x^{4}\right\rangle \geq \ldots$ becomes stationary at some point, hence there exist $n \in z^{+}$and $p(x) \in Z[X]$ for which $x^{n}=x^{n+1} p(x)$; and since this last condition is obviously satisfied by nilpotent elements as we11, a result of Chacron ([3], [2, Theorem 1]) shows that $R$ is periodic, hence contains non-zero idempotents. The following lemma gives crucial information about the idempotents.

LEMMA. If $\mathrm{R}$ satisfies the hypotheses of Theorem 2 and $\mathrm{e}$ is any non-zero idempotent, then $A_{r}(e)$ and $A_{\ell}(e)$ are finite.

PROOF. Assume without loss that $e$ is a left zero divisor; note that in any periodic ring, idempotents have finite additive order. Recall our initial remark, which implies that if $A_{r}(e)$ is infinite, it must contain an infinite zero ring.

Let $B$ be any zero ring contained in $A_{r}(e)$, and let $u$ be an arbitrary element of B. Considering the chain $\langle e, u\rangle \geq\langle e, 2 u\rangle \supseteq\langle e, 4 u\rangle \geq \ldots$ yields $k \in \mathrm{z}^{+}$such that $2^{\mathrm{k}} \mathrm{u} \in\left\langle\mathrm{e}, 2^{\mathrm{k}+1} \mathrm{u}\right\rangle$ - that is, there exist $\mathrm{p}, \mathrm{q}, \mathrm{t} \in \mathrm{Z}$ such that

$$
2^{k} u=p e+q 2^{k+1} u+t 2^{k+1} u e .
$$

Left-multiplying by e yields pe=0, hence $\left(2^{k}-q 2^{k+1}\right) u=t 2^{k+1}$ ue, and the fact that $e$ has finite additive order shows that $u$ does also. We now know that any subring $E$ of $R$ generated by $e$ and a finite number of elements of $B$ is finite. Choosing a maximal $E$, say $E_{1}$, and noting that $B \subseteq E_{1}$, we see that $B$ is finite. The proof of the lemma is now complete.

Returning to the proof of Theorem 2, suppose that e is an idempotent which is a zero divisor, say a left zero divisor; and write $R=e R+A_{r}(e)=e R e+\left(e R \cap_{A_{\ell}}(e)\right)+A_{r}(e)$. The last two summands are finite by the lemma, and the first is a ring satisfying our 
original hypotheses and having a multiplicative identity element. Of course, if all idempotents of $R$ are regular, then $R$ has a multiplicative identity element; therefore, we have reduced the problem to proving the theorem under the additional hypothesis that $R$ has 1 , in which case the periodicity of $R$ implies that $R$ has non-zero characteristic.

If there exists a non-zero idempotent $f \neq 1$, the decomposition $R=f R+(1-f) R$ shows that $R$ is finite, since both summands are finite by the lemma. Therefore, assume that 1 is the only non-zero idempotent, and use the perfodicity of $R$ to obtain the property that every element is either nilpotent or invertible - a property that forces $\mathrm{N}$ to be an ideal [7]. The factor ring $\frac{R}{N}$ has ascending chain condition and descending chain condition on subrings, hence is finite by (I). Now consider $N$, and let $B_{1}$ be any zero ring contained in $N$. Among subrings of $R$ generated by 1 and finitely many elements of $B_{1}$, choose $M$ to be a maximal one. Note that $M$ is finite and $B_{1} \subseteq M$; hence $B_{1}$ is finite, $N$ is finite, and $R$ is finite.

3. A THEOREM ON PERIODIC RINGS.

The final theorem may be thought of as an extension of Herstein's result ([6], [2, Theorem 2]) that periodic rings with $N$ C are necessarily commutative.

THEOREM 3. Let $R$ be a periodic ring having only finitely many non-central zero divisors. Then $R$ is either finite or commutative.

PROOF. Let $n(R)$ denote the number of non-central zero divisors, and note that Herstein's result implies commutativity of $R$ if $n(R)=0$. Assume henceforth that $n(R) \geqq 1$; and consider first the case that every element of $R$ is a left zero divisor or, more generally, the case that the set $D$ of left zero divisors is a non-trivial additive subgroup of $R$. Then for $d \in D \backslash C$ and $u \in D \cap C, d+u \in D \backslash C$; hence $\{d+u \mid u \in D \cap C\}$ is finite. Thus, $D$ is finite; and $R$ is finite by (II). This argument covers the case $R=N$, so we assume that $R \neq N$ and therefore $R$ contains non-zero idempotents.

If every non-zero idempotent is regular, there exists a unique non-zero 1 dempotent, necessarily 1; and every element is invertible or nilpotent. It follows, again by [7], that $\mathrm{N}$ is an ideal; and since $\mathrm{N}$ is equal to the set $\mathrm{D}$ of left zero divisors, $R$ is finite.

Assume now that we have a counterexample $R$ with $n(R)$ as small as possible. Then there exists $y \notin D$ and therefore an idempotent $e \notin D$. Thus $R$ has a left identity element; and since we can repeat our previous arguments for right zero divisors, $R$ has a right identity as we11, hence $R$ has 1 . Moreover, by the argument in the previous paragraph, $R$ has an idempotent $e$ which is a zero divisor. If $e \notin c$, then at least one of $\mathrm{eR}$ and $\mathrm{Re}$ must be non-commutative. On the other hand, if e $\epsilon \mathrm{C}$, then $\mathrm{R}=\mathrm{e} \mathrm{R} \oplus(1-\mathrm{e}) \mathrm{R}$, where $\oplus$ denotes a ring-theoretic direct sum; and since $\mathrm{R}$ was a counterexample, one of the summands must be non-commutative. Thus, in any event we may assume eR to be non-commutative.

Now eR must contain a non-central element $d$ which is a left zero divisor in eR; otherwise, eR would be commutative by Herstein's result. For $u=(1-e) x \in C \cap(1-e) R$, we have eu=ue=0, hence $u$ left-annihilates $e R$ and $d+u$ is a non-central left zero divisor in $R$. Thus, $C \cap(1-e) R$ is finite; and since (1-e)R consists of zero divisors 
in $R$, it contains only finitely many elements not in $C$, hence must be finite. Now eR cannot be finite as well, since $R=e R+(1-e) R$; therefore $n(e R)=n(R)$, and every non-central zero divisor in $R$ must be a zero-divisor in eR. It follows that $(1-e) R \subseteq C$. But then for any non-central zero divisor $d$ and any element $u \in(1-e) R$, $d+u$ is a non-central zero divisor, so both $d$ and $d+u$ are in $e R$ and therefore $u \in$ eR. But this implies $(1-\mathrm{e}) \mathrm{R}=\{0\}$, which is a contradiction. This completes the proof. 4. REMARKS.

In Theorem 3 the hypothesis of finitely many non-central zero divisors cannot be replaced by the assumption that $R$ has only finitely many non-central nilpotent elements. A counterexample is the direct sum $\mathrm{F} \oplus \mathrm{s}$, where $\mathrm{F}$ is an infinite periodic field and $S$ is a finite non-commutative nil ring.

A plausible extension of the Putcha-Yaqub result - namely, that a ring $R$ having only a finite number of regular elements must either be finite or consist entirely of zero divisors - is also false, even for commutative rings. To see this, consider the algebra $A$ over $G F(2)$ having basis $\left\{1, e_{1}, e_{2}, \ldots, e_{n}, \ldots\right\}$, where the $e_{i}$ are pairwise orthogonal idempotents. Certainly $A$ is not finite, and it is easily shown that 1 is the unique regular element.

ACKNOWLEDGEMENT. Supported by the Natural Sciences and Engineering Research Council of Canada, Grant No. A3961.

\section{REFERENCES}

1. BELL, H.E. Infinite Subrings of Infinite Rings and Near-rings, Pacific J. Math. 59 (1975), 345-358.

2. BELL, H.E. On Commutativity of Periodic Rings and Near-rings, Acta Math. Acad. Sci. Hungar. 36 (1980), 293-302.

3. CHACRON, M. On a Theorem of Herstein, Canad. J. Math. 21 (1969), 1348-1353.

4. GANESAN, N. Properties of Rings with a Finite Number of Zero Divisors, Math. Ann. 157 (1964), 215-218.

5. GANESAN, N. Properties of Rings with a Finite Number of Zero Divisors II, Math. Ann. 161 (1965), 241-246.

6. HERSTEIN, I.N. A Note on Rings with Central Nilpotent Elements, Proc. Amer. Math. Soc. $\underline{5}(1954), 620$.

7. OUTCALT, D. and YAQUB, A. A Commutativity Theorem for Rings, Bull. Austral. Math. Soc. 2 (1970), 95-99.

8. PUTCHA, M.S. and YAQUB, A. Rings with a Finite Set of Nonnilpotents, Internat. J. Math. \& Math. Sci. 2 (1979), 121-126.

9. SZELE, T. On a Finiteness Criterion for Modules, Pub1. Math. Debrecen 3 (1954), 253-256. 


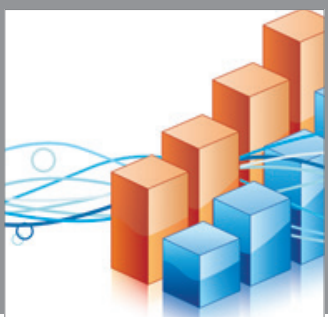

Advances in

Operations Research

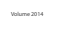

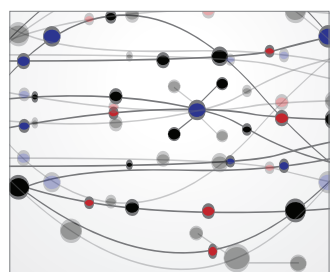

\section{The Scientific} World Journal
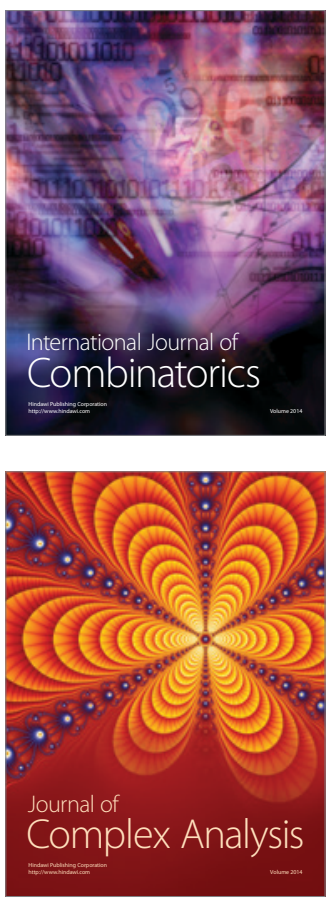

International Journal of

Mathematics and

Mathematical

Sciences
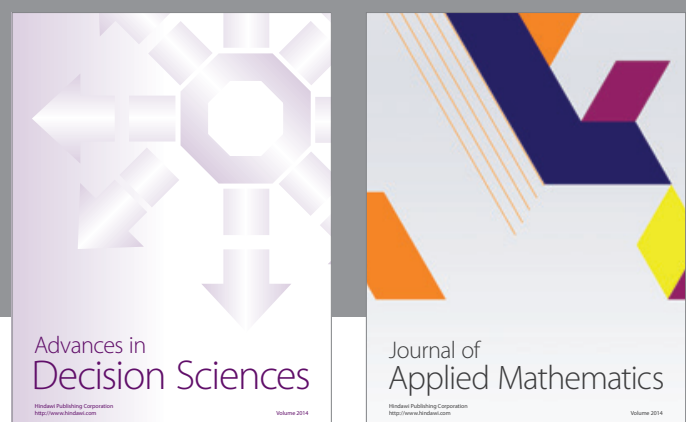

Journal of

Applied Mathematics
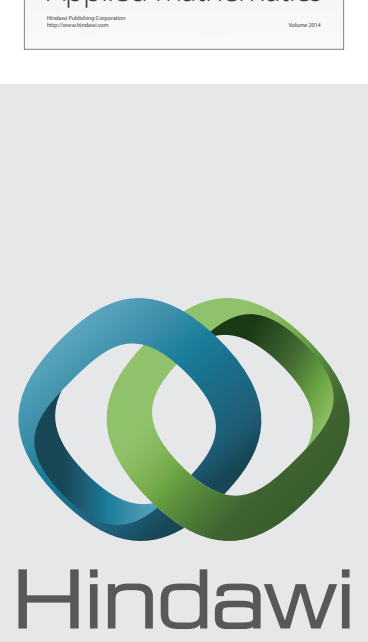

Submit your manuscripts at http://www.hindawi.com
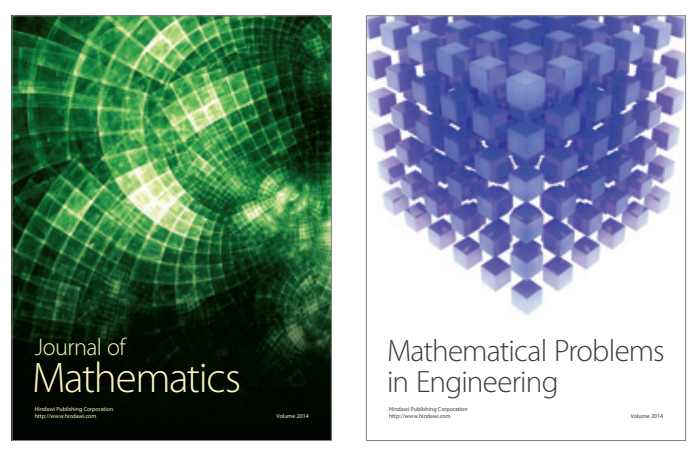

Mathematical Problems in Engineering
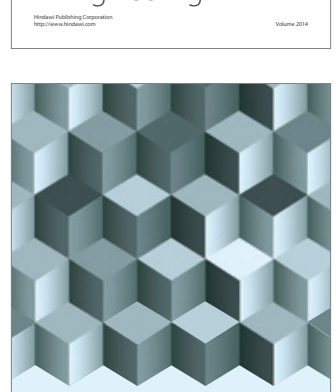

Journal of

Function Spaces
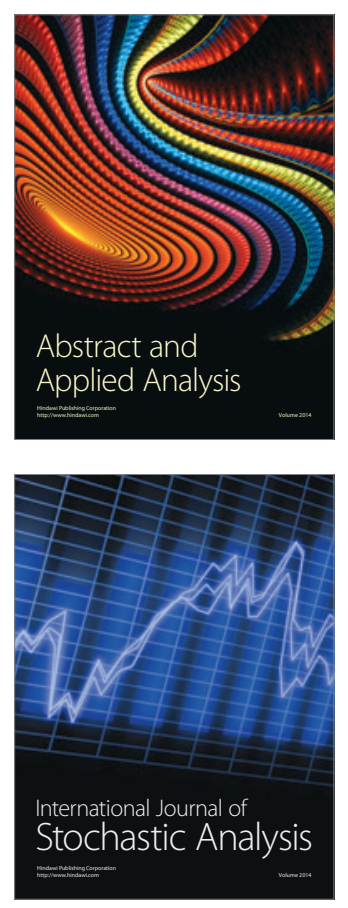

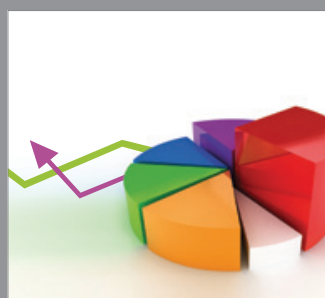

ournal of

Probability and Statistics

Promensencen
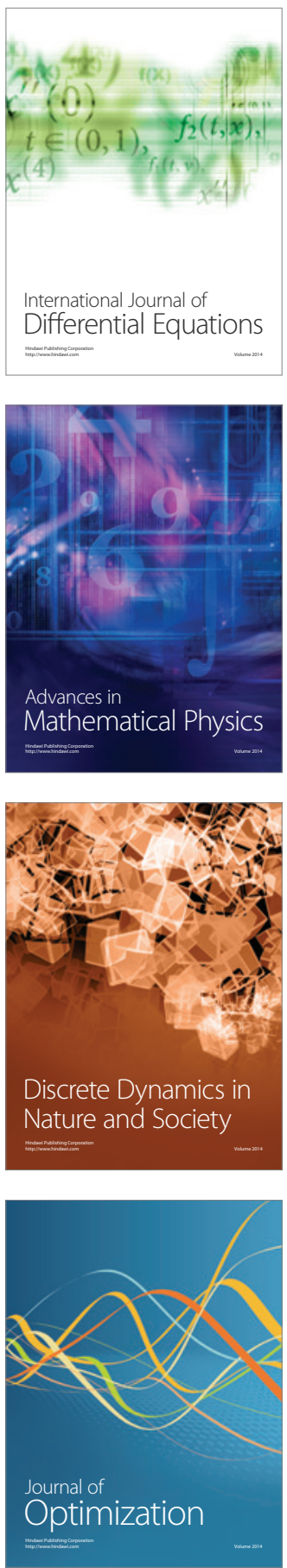